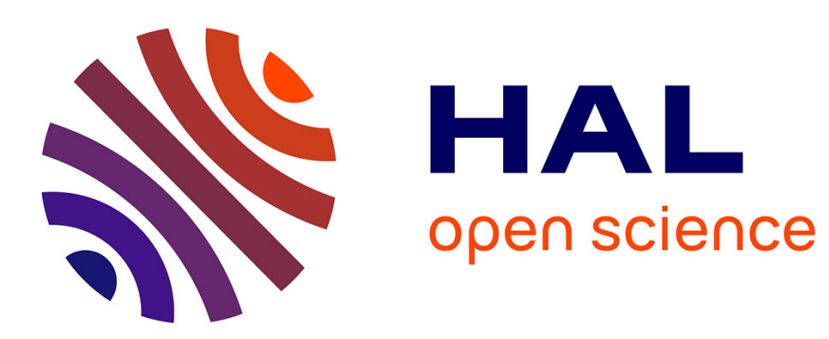

\title{
Recherche et non-recherche. Les valeurs à l'oeuvre dans l'évaluation des protocoles épidémiologiques
}

Nicolas Lechopier

\section{To cite this version:}

Nicolas Lechopier. Recherche et non-recherche. Les valeurs à l'oeuvre dans l'évaluation des protocoles épidémiologiques. Epidemiology and Public Health = Revue d'Epidémiologie et de Santé Publique, 2010, 58 (1), pp.41 - 48. 10.1016/j.respe.2009.09.006 . halshs-01638143

\section{HAL Id: halshs-01638143 \\ https://shs.hal.science/halshs-01638143}

Submitted on 19 Nov 2017

HAL is a multi-disciplinary open access archive for the deposit and dissemination of scientific research documents, whether they are published or not. The documents may come from teaching and research institutions in France or abroad, or from public or private research centers.
L'archive ouverte pluridisciplinaire HAL, est destinée au dépôt et à la diffusion de documents scientifiques de niveau recherche, publiés ou non, émanant des établissements d'enseignement et de recherche français ou étrangers, des laboratoires publics ou privés. 


\title{
Recherche et non-recherche. Les valeurs à l'oeuvre dans l'évaluation des protocoles épidémiologiques
}

\section{Research and non-research. The values in the evaluation of epidemiological protocols}

\author{
N. Lechopier ${ }^{1}$ \\ Institut d'histoire et de philosophie des sciences et des techniques, 13, rue du Four, 75006 Paris, France \\ Reçu le 7 avril 2009 ; accepté le 18 septembre 2009 \\ Disponible sur Internet le 29 janvier 2010
}

\begin{abstract}
Background. - Evaluation and ethical review of epidemiological research projects raises the problem of the limits between research and nonresearch. This ambiguous boundary reflects the status of this discipline at the crossroads between research and practical action. The question then is: in the field of health research, what gives data collection and analysis its quality of scientific activity?

Methods. - A conceptual and empirical study has been conducted about the practices of epidemiological research evaluation, centred on the case of the French Consultative Committee for the data processing in health research (CCTIRS), which is a consultative board that permits the National commission for the personal data protection (CNIL) to take decision about health research protocols that process personal data. The study was realized from 2003 to 2006 .

Results. - It is shown that the evaluation of such research protocols processing personal data articulates intimately two kinds of criteria: methodology and relevance. By studying and characterizing the different kinds of protocols that are judged not to be "scientific research" (poor science, pseudo-science and non-science), it becomes possible to understand the motives that lead to distinguish between what is and what is not research in epidemiology. A special attention is given to two kinds of problematic cases: firstly, the case of conflict of interests into the protocols themselves (i.e. seeding trials or surveys); secondly, the problem of epidemiological registers and other databases which are not hypothesisoriented. This last case leads to relate the conceptual frame of the committee with historical circumstances (the way which this discipline was introduced in France) and also mere epistemological considerations (the question of induction and generalizability).

Conclusion. - The activity of this committee illustrates a differentiated conception of what is research in epidemiology, influenced by explanatory analytical research paradigms. Finally, the field of epidemiological research appears to be structured by some values that appear through the elaboration and the application of the ethical and regulatory texts.
\end{abstract}

(C) 2009 Elsevier Masson SAS. All rights reserved.

Keywords: Conflict of interest; Epidemiological study characteristics; Confidentiality; Ethics; Research; Peer review

\section{Résumé}

Position du problème. - L'évaluation des projets de recherche épidémiologique et leur révision éthique soulève le problème de la limite entre recherche et non-recherche. Cette frontière ambiguë reflète le statut de cette discipline, au carrefour de la recherche et de l'action. La question est donc ici : dans le champ de la recherche en santé, qu'est-ce qui fait de la collecte et de l'analyse de données une activité à valeur scientifique ?

Méthodes. - Une enquête empirique et conceptuelle a été réalisée en prenant pour objet les pratiques d'évaluation des protocoles de recherche épidémiologique. Elle est centrée sur le cas du Comité consultatif sur le traitement de l'information en matière de recherche dans le domaine de la santé (CCTIRS), qui donne des avis consultatifs permettant à la Commission nationale informatique et libertés (CNIL) de se prononcer sur ces projets de recherche qui traitent des données à caractère personnel. Cette enquête a été réalisée entre 2003 et 2006.

Résultats. - L'appréciation des protocoles de recherche en santé traitant des données personnelles articule étroitement des critères méthodologiques et des considérations liées à la pertinence des études. En présentant les différents types de protocoles qui sont jugés comme n'entrant pas dans le cadre de la recherche scientifique (mauvaise science, fausse science et non-science), on met en lumière les motifs pour lesquels est distingué en épidémiologie ce qui relève de la recherche scientifique et ce qui n'en relève pas. Deux cas limites sont particulièrement

\footnotetext{
Adresse e-mail : nicolas.lechopier@laposte.net.

${ }^{1}$ Rattachement institutionnel : université Lyon-1.
} 
discutés : les conflits d'intérêts intrinsèques aux protocoles (enquêtes promotionnelles ou seeding trials) et le cas des registres et bases de données sans hypothèses directrices. Ce dernier cas conduit à rapporter le cadre conceptuel de l'activité du comité à des facteurs historiques (renvoyant au contexte à travers lequel cette discipline a été introduite en France) et épistémologiques (la question de l'induction et de la généralisabilité).

Conclusion. - L'activité du comité illustre une conception identifiable de la nature de la recherche en épidémiologie, influencée par les paradigmes de la recherche explicative analytique. En définitive, la recherche en épidémiologie apparaît structurée autour de certaines valeurs qui apparaissent en creux dans l'élaboration et l'application des textes éthiques qui l'encadrent.

(C) 2009 Elsevier Masson SAS. Tous droits réservés.

Mots clés : Conflit d'intérêts, Caractéristiques d'une étude épidémiologique comme sujet, Confidentialité, Éthique de la recherche, Évaluations par les pairs

L'épidémiologie est une discipline profondément « hétérogène dans ses objectifs, ses méthodes, ses pratiques » [1]. L'autonomie qu'elle revendique comme science ou comme discipline de recherche s'avère d'ailleurs souvent problématique [2]. À l'Inserm aujourd'hui, l'épidémiologie fait partie de la « recherche en santé », ce qui la tient relativement à l'écart de la « recherche médicale », alors que son activité de surveillance épidémiologique contribue encore à accentuer son particularisme [3-5]. L'épidémiologie est aussi tiraillée entre plusieurs familles disciplinaires : à l'université, elle est une discipline médicale rattachée à la santé publique mais pourrait aussi bien l'être à la statistique [6]. En outre, les épidémiologistes publient principalement en dehors des revues d'épidémiologie : une analyse bibliométrique estimait que deux tiers des publications de directeurs et directrices d'unité et d'équipes Inserm en épidémiologie, sur la période 2001-2002, étaient parues dans des revues généralistes scientifiques et médicales ou des journaux spécialisés, pour seulement un tiers dans des revues d'épidémiologie ou de biostatistiques [7]. Tout se passe comme si l'épidémiologie peinait à trouver sa juste place dans le paysage institutionnel et disciplinaire. Est-elle la « discipline scientifique de base » ou une branche de la santé publique [2] ? Est-elle une science produisant par des recherches spécifiques des connaissances qui lui sont propres ou un corpus de méthodes quantitatives au service d'autres disciplines [8] ?

Ces hésitations concernant l'identité professionnelle et académique de l'épidémiologie - qui ne sont ni nouvelles ni seulement françaises - posent des problèmes peut-être plus profonds au regard du système de régulation éthique de la recherche [9]. Par exemple, la loi du $1^{\text {er }}$ juillet 1994 qui définit en France les conditions dans lesquelles une recherche scientifique dans le domaine de la santé peut légitimement collecter des données à caractère personnel. Ces conditions se rapportent au respect de l'autonomie des personnes, à la minimisation des risques de rupture de la confidentialité ainsi qu'à la valeur du projet de recherche lui-même. Mais qu'appelle-t-on recherche dans le domaine épidémiologique ? À partir de quand, jusqu'où et pour quelles raisons la collecte des données sur des questions de santé est-elle une activité scientifique, susceptible alors de bénéficier des droits (par exemple, l'accès à des données sensibles) accordés à cette fonction ? Cet article s'appuie sur une étude des pratiques de révision de la recherche épidémiologique qui renouvelle le traitement de cette question, telle qu'elle s'est posée notamment aux États-Unis [10,11]. Partant d'une thèse philosophique selon laquelle les activités scientifiques ne sont pas sans référence aux valeurs en particulier dans le cas de l'épidémiologie [12-15], nous suggérons que la distinction entre recherche et «non-recherche » dépend non seulement des objets et des méthodes employées mais aussi des valeurs, celles-là même qui servent de base à l'évaluation des protocoles.

\section{Le cas du Comité consultatif sur le traitement de l'information en matière de recherche dans le domaine de la santé}

Une enquête consacrée aux conditions dans lesquelles les chercheurs peuvent faire usage de données personnelles dans leurs protocoles, telles que définies dans la loi du $1^{\text {er }}$ juillet 1994 a été réalisé, entre 2003 et 2005 [16-18]. Nous nous sommes en particulier penchés sur l'activité du Comité consultatif sur le traitement de l'information en matière de recherche dans le domaine de la santé (CCTIRS), rattaché au ministère de la Recherche et composé de personnes compétentes en matière de recherche dans le domaine de la santé, de l'épidémiologie, de la génétique et de la biostatistique. Ce comité a pour rôle d'émettre un avis sur la méthodologie de la recherche au regard des dispositions de la loi « informatique et libertés » du 6 janvier 1978, sur la nécessité du recours à des données à caractère personnel et la pertinence de celles-ci par rapport à l'objectif de la recherche, préalablement à la saisine de la Commission nationale de l'informatique et des libertés (CNIL).

Institution unique dans le paysage des comités régulateurs de la recherche, le CCTIRS fonctionne simultanément comme un comité scientifique et de fait comme un comité d'éthique. Il est en premier lieu un comité scientifique d'évaluation de la qualité des projets de recherche. Son avis est consultatif, mais il s'inscrit légalement dans un circuit d'autorisation administrative, puisqu'il précède et contribue largement à la décision de la CNIL. Ce comité est composé de 15 membres, diplômés en épidémiologie ou biostatistique, le plus souvent en complément d'une spécialité médicale (clinique ou santé publique). Bien qu'aucun membre de la société civile n'y siège et que le mot « éthique » ne soit pas présent dans le texte de loi qui le régit, c'est quand même un comité ayant des compétences dans le champ de l'éthique puisqu'il évalue a priori la façon dont le responsable d'une recherche compte prévenir les risques d'atteinte à la confidentialité de la vie privée des participants. Alors que les comités de protection des personnes (CPP) relevant de la loi du 20 décembre 1988 sont chargés uniquement des recherches biomédicales interventionnelles, le CCTIRS est chargé d'évaluer également les projets purement observationnels. C'est probablement pour éviter les conflits entre ces deux 
instances que le second n'a pas été explicitement constitué comme un comité de révision éthique de la recherche, ce qui a d'ailleurs généré des problèmes de validation au regard des standards internationaux, récemment soulignés [19-20]. Dans la perspective prochaine d'une réorganisation du système français d'encadrement de la recherche sur l'être humain, sa place et sa fonction à venir demeurent encore incertaines (discussion d'une proposition de loi $\mathrm{n}^{\text {os }} 1372$ et 1377 relative aux recherches sur la personne, Assemblée nationale, 22 janvier 2009).

Nous avons participé régulièrement aux réunions mensuelles du CCTIRS entre 2003 et 2005 pour étudier la façon dont les protocoles étaient évalués par le comité. Cette enquête de terrain a été complétée par une série d'entretiens avec des experts membres et de personnalités extérieures (auquel nous nous référons ici en utilisant la notation Ent + numéro d'anonymat) ainsi qu'une étude quantitative sur la variété des dossiers et la répartition des avis - analyse ayant nourri le rapport annuel 2006. Les observations rapportées ici ne concernent que la façon dont le comité fonctionnait durant cette période d'activité, mais les analyses qu'elles soutiennent vont probablement un peu au-delà.

En 1998, le comité a adressé au président de la CNIL un texte visant à préciser ce qui orientait son activité d'évaluation. Ce court texte a déjà été publié dans ces colonnes [21] :

Le Comité consultatif ne se prononce pas sur l'intérêt particulier que peuvent avoir des résultats pour les promoteurs des études qui lui sont soumises pour avis. Seuls les résultats obtenus dans le cadre d'une recherche ont un intérêt général pouvant justifier le recours à des données nominatives.

Deux critères primordiaux définissent une recherche :

- la formulation d'objectifs et d'hypothèses ;

- la mise en œuvre d'une méthodologie permettant d'atteindre ces objectifs ou de tester ces hypothèses.

Ce travail doit tendre à l'amélioration des connaissances.

Dans le domaine de la santé, la recherche doit faire appel à des techniques d'observation validées, décrites avec précision dans le protocole (questionnaires, échelles, critères de diagnostic...) et les groupes étudiés doivent être représentatifs. La nature et la quantité des informations recueillies dans une base de données ne suffisent pas à définir une recherche. Le fait qu'une étude porte sur 5000 malades ou 1000 médecins et qu'un questionnaire ait 300 items ne permet en aucun cas de supposer que les conditions énoncées ci-dessus sont remplies.
Cette mise au point est riche mais dessine aussi une conception spécifique de ce qu'est la recherche en épidémiologie. Quels sont les fondements épistémologiques de ces critères de définition? Comment fonctionnent-ils en pratique ?

\section{L'évaluation des protocoles de recherche et les cas de disqualification}

Selon le texte de loi qui l'institue, le CCTIRS a pour fonction de donner un avis sur la méthodologie des recherches. Ses membres sont qualifiés par leur compétence en méthodologie. Du point de vue des spécialités représentées (qui vont de la génétique à la santé au travail, en passant par la cancérologie, l'économie de la santé, etc.), le comité est assez hétérogène, mais il est relativement homogène au plan du rattachement professionnel : la quasi-totalité des experts travaillent comme praticiens et chercheurs dans des institutions publiques (centres hospitalo-universitaires, établissements publics scientifique, agences, etc.) et aucun ne travaille à plein temps pour l'industrie. La procédure mobilise deux experts qui évaluent les objectifs explicites du projet, la justification du nombre de sujets nécessaire, les critères de jugement, les cahiers d'observation, etc. Il s'agit de savoir si la méthodologie utilisée est cohérente par rapport aux objectifs $\mathrm{du}$ projet et si le protocole est effectivement susceptible d'apporter une réponse à la question posée. Ces experts consignent leurs remarques dans des rapports qui sont lus en séance. Après discussion générale, le comité donne un avis favorable, réservé ou défavorable. Dans la suite, nous insisterons d'abord sur les critères qui orientent la production des avis du comité, puis nous nous intéresserons aux cas de disqualification de certains dossiers comme n'étant « pas de la recherche ».

\subsection{L'articulation complexe des critères d'expertise : qualité méthodologique et pertinence}

Dans le texte explicatif du travail du comité reproduit cidessus, le terme « objectif » désigne ce qui est visé par la recherche, la nature et le genre de résultats auxquels on veut parvenir, tandis que le terme « hypothèse » renvoie à un concept plus précis et spécifique. Les objectifs se divisent en principaux (la question de recherche) et en « secondaires » (les questions subsidiaires, souvent descriptives). Prouver l'efficacité d'un traitement, évaluer l'impact d'une méthode de prise en charge clinique, estimer la prévalence de tel ou tel facteur de risque dans une population donnée sont des objectifs principaux. Les objectifs secondaires sont par exemple : décrire le profil des patients répondants, estimer les coûts induits par l'usage d'un traitement, etc. L'hypothèse peut être de supériorité, d'équivalence, de non-infériorité, etc. Il faut qu'elle soit précisément définie si l'on veut s'assurer qu'au moment de l'analyse des résultats les données permettront de la rejeter ou pas. Le terme « objectif » est méthodologiquement neutre, tandis que « hypothèse » renvoie à un schéma déductif : les données pertinentes sont celles qui vont permettre de tester cette hypothèse. 
Dans ce texte, aucune qualification n'est apportée au terme " hypothèse ». Il n'est en effet pas nécessaire que cette hypothèse soit par exemple "fondée », " surprenante », « originale », etc. Il est simplement demandé qu'une hypothèse soit présente et opérante. C'est la pierre de touche qui permet au protocole de produire de la connaissance. Cependant, les experts ne s'en tiennent pas seulement à cette considération formelle. "Quand sur le fond d'un dossier je suis compétente, mon côté expertise scientifique ressort, je suis plus méchante quand la question n'est pas bien intéressante. Mais j'essaie de m'abstraire de ça et de regarder ce qu'on me demande de regarder, c'est-à-dire la méthodologie. Je regarde quand même un peu la pertinence de la question parce que ce ne sera pas un argument-clé, mais si c'est particulièrement mauvais et pas tellement pertinent, j'aurai envie de dire... non non là vraiment...»(Ent5). Ce témoignage conforte l'observation que des considérations de « pertinence » peuvent entrer en ligne de compte et jouer un rôle modulateur dans le processus d'évaluation pourtant centré sur la " méthodologie ». Les experts évaluent la qualité méthodologique d'un projet non seulement pour elle-même mais aussi à l'aune du problème traité et de l'importance des résultats attendus. Pour une enquête sur la survenue de cas de cancers près d'une usine de retraitement de déchets, on jugera indispensable d'avoir la meilleure méthodologie possible et des critères de jugement adéquats. En revanche, pour une enquête sans grande nouveauté, la méthodologie devra être simple et efficace, parcimonieuse vis-à-vis des données. Ainsi, s'il est important de ne pas confondre jugement méthodologique et évaluation de l'intérêt d'une recherche [22], on peut penser qu'en réalité le niveau de qualité méthodologique exigé n'est pas tout à fait indépendant du contexte dans lequel une recherche s'inscrit.

Comment juge-t-on de la pertinence d'une enquête ? On peut distinguer trois sortes de justifications. Une recherche est pertinente si elle est inscrite dans le questionnement concret de praticiens et qu'elle est à même de produire des résultats opérationnels. La pertinence signifie ici l'utilité, l'applicabilité ou la capacité à éclairer des problématiques pratiques concrètes. Que va changer la réponse à cette question ? Quelles sont les « retombées attendues » (Ent14) de cette recherche ? Deuxièmement, la pertinence d'une recherche est son importance « humanitaire ». Le dépistage du saturnisme chez les enfants et les femmes enceintes ou allaitantes à proximité d'un site industriel pollué constitue une recherche importante. L'étude du profil du consommateur d'un médicament dit « de confort» l'est beaucoup moins. Tout cela est discutable à cause du « biais compassionnel » que ces considérations peuvent produire. C'est cependant un fait que, au sein du comité, un accord tacite se forme aisément sur ce qui est important ou non en termes de santé publique. Troisièmement, la pertinence s'entend aussi au sens épistémique. Les résultats auront-ils un degré suffisant de généralisation possible pour pouvoir éclairer des situations multiples, au-delà des situations qui ont suscité la question ou servi à fournir cette réponse ? Cette troisième acception de la pertinence met l'accent sur le fait qu'ils devront constituer d'authentiques connaissances scientifiques. Une recherche est pertinente lorsqu'elle vient « combler un manque dans nos connaissances » (Ent17), autrement dit lorsqu'elle peut représenter un progrès, un renouvellement théorique ou méthodologique significatif.

L'épidémiologie se présente ici comme une pratique de recherche empirique utilisant des méthodes statistiques et d'inférence probabiliste, conduisant des recherches appliquées (elle doit être opérationnelle), traitant de problèmes humains (elle se préoccupe de fléaux) et produisant des faits scientifiques (elle contribue au progrès de la connaissance). Pragmatisme, lutte contre la maladie et savoir scientifique sont les trois sphères de «justification » au sein desquels se déploie l'épidémiologie [23]. C'est sur ces trois dimensions d'une recherche que repose l'évaluation de la qualité proprement méthodologique du protocole. Il ne suffit pas seulement qu'un protocole emploie correctement des outils méthodologiques validés - bien qu'il arrive que cela soit suffisant. Celui-ci doit être valable, en adéquation avec l'usage prévu des résultats, bien positionné au regard de ces trois sphères de justification qui constituent le socle de la discipline épidémiologique, ses valeurs de base.

\subsection{Ce qui n'est pas de la recherche}

Un phénomène va nous conduire à préciser ce que l'on entend par «connaissance » en épidémiologie. Pendant la période d'enquête sur le CCTIRS, certains dossiers étaient considérés comme «ne constituant pas une recherche ». Cette disqualification concernait environ $20 \%$ des dossiers (sur un total annuel moyen de 596 dossiers examinés entre 20032005). Jusqu'en 2006, ce jugement était associé à une décision d'irrecevabilité, autrement dit un avis ni favorable ni défavorable («cela n'est pas de la recherche ») devant laquelle la CNIL et les demandeurs d'avis n'avaient plus qu'à prendre une décision. Désormais, ce genre de décision est moins fréquent (le comité donne aujourd'hui davantage d'avis défavorables que lors de la période étudiée) et s'exprime dans une formulation légèrement différente. Les dossiers concernés sont loin de constituer une catégorie homogène, mais on peut distinguer deux problèmes bien différents.

\subsubsection{Mauvaise science et fausse science}

Un protocole est disqualifié lorsque « la méthode employée n'est pas adaptée à l'objectif énoncé et quand le recours à une méthodologie pertinente supposerait une refonte telle du projet qu'il s'agirait en définitive d'un autre projet » (Ent14). Soit un projet qui prétend étudier l'efficacité d'un dispositif médical en s'appuyant sur une méthodologie sans groupe de référence. L'expertise, dans ce cas, repose sur des « standards » méthodologiques : une preuve d'efficacité nécessite au minimum une comparaison entre un groupe traité et un groupe non-traité, avec un contrôle de différents biais. Cette recherche n'atteint pas les standards qui permettraient par exemple d'en publier les résultats dans une revue à comité de lecture. L'existence de ce genre de protocoles mal conçus tient au fait que l'épidémiologie offre un ensemble de méthodes qui sont utilisées par des acteurs (travailleurs sociaux, agents de santé, 
etc.) qui, tout en s'interrogeant sur la prévalence d'une affection ou l'efficacité d'un programme de prévention par exemple, ne sont pas nécessairement eux-mêmes des épidémiologistes professionnels. Certains protocoles sont alors considérés comme « honnêtement mauvais », disqualifiés pour leur déficience méthodologique et non à cause des intentions qui les orientent.

Un autre cas de figure se présente, inversé comme en miroir par rapport au précédent. «Le plus souvent, explique un expert, les membres du comité aident des collègues à améliorer leurs projets. Mais il est vrai que si ce n'est pas de la recherche au départ, on ne peut guère prétendre améliorer la méthodologie » (Ent2). Que signifie « être de la recherche au départ ?» Certains projets de recherche passant devant le comité sont tout simplement des «faux », comme l'on parle d'un faux tableau ou d'une fausse monnaie. Typiquement, il s'agit de projets qui peuvent apparemment être bien conçus, employer correctement des outils méthodologiques valides, mais qui en réalité poursuivent un but qui les situe en dehors de la sphère de la recherche scientifique - principalement celui de contribuer à la vente d'un produit en faisant de la publicité déguisée. Il peut y avoir, certes, des études de marché conduites rigoureusement, dans le but de connaître les indications pour lesquelles le produit est effectivement prescrit, le profil des consommateurs, etc. Mais certaines enquêtes ou études conduites par les laboratoires ont pour objectif principal - sinon exclusif - de contourner la loi dite « anti-cadeau » (Art. L.365-1 du Code de la santé publique, loi du 27 janvier 1993). Les questionnaires et cahiers d'observation sont distribués aux médecins, non pas pour réunir des données permettant de répondre à une question, mais pour couvrir une rémunération incitative qui serait illégale hors de cette supposée finalité de recherche ou d'évaluation scientifique. C'est le problème dit des seeding trials (pour la recherche clinique) ou plutôt ici des seeding surveys (pour le domaine pharmacoépidémiologique) dans lesquelles une logique purement commerciale se cache derrière une apparence de recherche. Ce genre de falsification a étrangement fait l'objet de très peu d'analyses spécifiques, mais la documentation d'un cas récent illustre bien la difficulté d'en saisir le caractère factice [24-27].

Ces faux protocoles de recherche épidémiologique imitent parfois très bien les vrais, mais ils sont caractérisés par une construction inverse. Le recueil de données n'est pas défini en fonction de l'objectif, comme cela devrait être le cas pour toute recherche authentique. Au contraire, l'objectif directeur du protocole est construit a posteriori afin de légitimer la mise en place d'une logistique incitant à la prescription du produit. Comme ce genre de projet n'est pas rare, les membres du comité acquièrent au cours du temps le savoir-faire pour débusquer ces pseudo-recherches. La construction inverse se repère principalement dans le mode de calcul du nombre de sujets nécessaire - des analyses en sous-groupe permettent de gonfler artificiellement l'effectif de l'échantillon - ou dans la détermination de la différence attendue - les auteurs des protocoles fixent des exigences disproportionnées de précision afin d'augmenter la quantité de données requises. Pour révéler la présence d'un tel conflit d'intérêts au cœur de la production de la recherche, les experts s'appuient également sur des éléments liés au statut du demandeur en regardant par exemple s'il s'agit du laboratoire commercialisant le produit concerné ou une entreprise contractualisée pour réaliser la recherche. Bien que l'expertise ne se résume jamais à des arguments $\mathrm{ad}$ hominem, il est clair que les références, la réputation et le style même de rédaction jouent un rôle dans la façon dont l'expert va aborder le projet dont il est rapporteur. Il arrive souvent que des dossiers suscitent la perplexité des experts : la frontière entre l'authentique et l'inauthentique en matière de recherche n'est pas aisée à tracer.

\subsubsection{La non-science : l'information sans visée de généralisation}

Les disqualifications prononcées par le CCTIRS soulèvent un autre problème de frontières, qui révèle d'autres valeurs à l'œuvre dans l'évaluation de la recherche. Durant la période 2002-2005, une bonne part des projets de bases de données et de registres, représentant au minimum $3 \%$ des dossiers annuellement examinés par le comité, soit une vingtaine de dossiers par an, se voyaient également disqualifiés comme n'étant pas à proprement parler de la recherche. Comme le dit explicitement le texte cité concernant le champ de compétence du comité, la quantité et le type de données ne sont pas suffisants pour définir une recherche, quand bien même ce recueil serait réalisé consciencieusement et suivant une «bonne intention ». Tant que l'on n'a pas formulé précisément d'hypothèse directrice, le risque est celui de « l'auberge espagnole » (Ent11), de la fishing expedition, ou encore d'avancer à l'aveuglette. S'il y a effectivement des bases de données qui obtiennent un avis favorable lorsque le modèle théorique sous-jacent est explicite, le choix de disqualifier la plupart des enregistrements de données non structurés autour d'une hypothèse à tester mérite d'être questionné ici. Cela permettra de préciser certaines valeurs proprement « épistémiques », c'est-à-dire liées au genre de connaissance produite, qui orientent l'évaluation de la recherche par le CCTIRS.

On peut partir d'une distinction élémentaire entre les études descriptives, qui visent à mesurer un état de santé dans une population donnée (Qui ? Où ? Quand ? Combien ?) des recherches analytiques qui fournissent des explications en contribuant à la recherche de relations de causalité (À cause de quoi ? Comment ?). Cette distinction se retrouve dès les tous premiers traités de méthodologie de l'épidémiologie moderne publiés en langue française : C. Rumeau-Rouquette et D. Schwartz considèrent dans la première édition des Méthodes en épidémiologie que celle-ci est « naturellement » divisée en deux types de démarches [28]. La démarche descriptive vise à énoncer des faits à propos de la population dont on n'a étudié, souvent, qu'un échantillon. L'attitude analytique au contraire part d'une observation ou d'une expérience réalisée sur un nombre limité de cas pour en tirer « une loi dont la validité serait supposée pour une infinité de situations qui débordent largement en nombre et en diversité celle qui a été expérimentée ou observée » [29]. Le terme de recherche est alors réservé à ces enquêtes contrôlées qui seules sont capables 
de fournir des explications en contribuant à déterminer quelles sont les causes ou les lois générales du phénomène étudié (la survenue d'une maladie par exemple). Dans un autre texte, D. Schwartz retrace schématiquement l'histoire des progrès de la méthode épidémiologique et présente le moment descriptif comme une première étape, préscientifique, ne mobilisant pas les outils de la statistique inférentielle et donc incapable de faire connaître les causes [30]. Mais si le moment descriptif est toujours nécessaire, pour mesurer l'importance des problèmes, générer des hypothèses et juger des " progrès accomplis », toutes les connaissances produites auront besoin d'être testées par un véritable protocole analytique [28]. La recherche analytique s'adosse à des hypothèses causales explicites que l'on entend tester. L'épidémiologie commence à être une science quand elle se préoccupe de connaître les causes et cherche ainsi à expliquer les données observées.

La mise au point qui précède permet de comprendre pourquoi le comité disqualifie comme n'étant pas de la recherche les bases de données qui ne sont pas structurées autour d'hypothèses précises. Cette position découle d'au moins deux sources d'ordres différents. Elle relève d'abord d'un positionnement épistémologique plus général dont le trait principal est la critique de la démarche inductive. Les inductivistes défendent l'idée que les connaissances scientifiques sont inférées, à partir des données observées, par un processus de généralisation empirique. Les détracteurs de cette démarche soulignent premièrement que l'induction ne peut pas fonctionner tout simplement parce qu'il est impossible de « recueillir tous les faits significatifs » si l'on ne connaît pas les hypothèses par rapport auxquelles ces données prendront leur signification » [31]. Une science ne peut pas fournir d'explications si elle ne se fonde pas sur des hypothèses. « Faute de telles hypothèses, analyse et classification sont aveugles ». Secondement, en toute logique, aucune théorie n'est jamais confirmée par des données empiriques, elle peut tout au plus être réfutée. La science procède donc rationnellement de façon déductive et non pas inductive.

Cette critique épistémologique des inférences inductives est renforcée par l'exigence de possibilité de généralisation, exigence que ne peuvent satisfaire les méthodes strictement descriptives. La généralisation doit être distinguée de l'extrapolation. Extrapoler, c'est conduire une inférence statistique à partir d'un échantillon vers une population dont il est (probablement) représentatif. Selon Rothman et Greenland, les résultats d'une extrapolation ne sont valables que pour les sujets qui auraient pu être sélectionnés lors de l'échantillonnage. Au contraire, pour ces auteurs, la généralisation va au-delà de l'extrapolation statistique et formule des concepts abstraits concernant les facteurs étudiés, « abstractions qui s'appliquent à un domaine d'expérience plus étendu que le domaine observé ou ayant servi à l'échantillonnage » [32]. Seules les démarches analytiques possèdent une telle validité externe, c'est-à-dire la propriété d'être valable pour d'autres populations en général, puisque fondées sur des comparaisons raisonnées visant à tester des hypothèses générales (comme «fumer des cigarettes cause le cancer du poumon »).
Voici le cadre conceptuel dans lequel le CCTIRS travaille : une conception déductiviste de la rationalité scientifique, qui va de pair avec une préférence donnée à l'épidémiologie à vocation explicative. Ce cadre trouve aussi sa source dans le mode de formation historique de l'épidémiologie moderne comme discipline. On connaît à cet égard l'importance de l'enquête inaugurale conduite en France par D. Schwartz sur la relation tabac-cancer [33,34]. Les méthodes de l'épidémiologie analytique ont été alors introduites en rupture avec une certaine conception de la santé publique de tradition hygiéniste. Comme le rappelle Gaudillière, «à une épidémiologie pensée comme discipline d'action accompagnant la montée en puissance des débats publics sur la santé et la politique du risque, Schwartz et ses collègues opposaient une épidémiologie visant moins à désigner des dangers ou à légitimer des interventions qu'à résoudre les problèmes rencontrés dans les jugements de causalité ou d'efficacité par des innovations procédurales et des investissements de forme. D'où, comme dans le cas de l'enquête tabac, un écart substantiel vis-à-vis des institutions et des débats de santé publique » [35].

Le comité a hérité de ce positionnement spécifique de l'épidémiologie moderne comme discipline d'élucidation des causes de maladies plutôt que comme simple outil d'information au service de la santé publique. Un recueil de données de santé n'a de valeur (scientifique) que par les hypothèses qui le structurent. Paradoxalement, la loi créant le CCTIRS a émergé à partir de pratiques d'épidémiologie à forte composante descriptive : les registres de morbidité mis en place en France à partir des années 1970 supposent de collecter des données sur tous les cas survenus dans une région donnée, ce qui implique une rupture de la confidentialité et du secret médical que la loi a $\mathrm{du}$ « couvrir » [37]. Hormis les registres légitimés par le Comité national des registres (qui conduisent effectivement des travaux de recherche), le CCTIRS a tendance à ne pas homologuer [36], comme « recherche », les bases de données qui se contentent d'enregistrer passivement des informations sans orientation théorique. Selon le domaine considéré, une approche inductive fondée sur de larges bases de données peut se justifier et être avalisée par le comité (en génomique ou en pharmacoépidémiologie par exemple). Mais tant que ces bases de données ne laissent entrevoir aucun débouché explicite en termes explicatifs ou cognitifs, il demande que lui soient soumis les protocoles de recherche qui dans le futur auront recours à cette base de données.

\section{Conclusions}

Le CCTIRS recourt à de multiples critères pour évaluer les protocoles de recherche, principalement la qualité méthodologique et la pertinence. Ces critères d'évaluation le conduisent à opérer, dans l'ensemble des projets de collecte de données relatives à la santé dans les populations, une démarcation, un partage entre ceux qui s'articulent correctement à un raisonnement probabiliste fondé sur des hypothèses et les autres, qui ne le font pas, ou pas correctement. Ces critères méthodologiques sont eux-mêmes modulés par des considérations liées à l'importance des problèmes traités, ce qui est 
normal dans un domaine de recherches appliquées aux problèmes de santé humaine, mais dont la justification relève d'une multitude de valeurs probablement irréductibles les unes aux autres. La façon de lier ces valeurs dans l'expertise de chaque dossier singulier n'est pas neutre. En considérant comme plus abouties les recherches qui s'intéressent aux causes par rapport à celles qui visent à établir un état des lieux de ces problèmes dans la population - ou plutôt telle ou telle population singulière - le comité tend à inscrire l'épidémiologie comme une discipline ayant pour valeur directrice la production d'explications causales. Certes, la connaissance objective des causes permet de fonder des actions de santé publique. En même temps, cette valeur directrice n'épuise probablement pas la dimension pragmatique et engagée de l'épidémiologie liée à son articulation avec la santé publique. Les controverses sur la scientificité, sur ce qui constitue ou non une recherche, reflètent en tout cas la riche ambivalence qui parcourt une discipline articulant savoir et action.

Lorsque le comité disqualifie un projet comme n'étant pas du domaine de la recherche, il le fait dans certains cas au regard de la qualité et de l'intégrité scientifiques. L'expérience du CCTIRS indique de façon inquiétante que, dans le champ de la recherche observationnelle en santé (mais le problème existe aussi dans la recherche clinique), il arrive assez fréquemment que les protocoles soient biaisés par des conflits d'intérêts structurels - sans même parler de ceux qui affectent la divulgation des résultats, lorsqu'il y en a. Le contrôle de la mauvaise science et de la fausse science, ou le refus d'homologuer ces protocoles comme « recherche scientifique », apparaissent comme des mesures cohérentes avec l'ambition de régulation éthique. En effet, il n'est pas éthique de demander à des personnes de donner « un peu de soi » [38] s'il s'agit de protocoles de mauvaise qualité ou (pire ?) de fausses recherches. Le problème n'est pas seulement que l'information et le consentement de la personne concernée seraient intrinsèquement faussés par cette situation ; il est aussi que l'on attend d'une recherche dans le domaine de la santé qu'elle soit orientée vers des buts « de recherche » et non un cheval de Troie de la force de vente. Cela ne concerne pas seulement la communauté des chercheurs mais aussi la société et les normes qu'elle produit pour fixer l'acceptable et l'inacceptable en matière de recherche sur l'être humain [39,40]. Depuis 20 ans en France, le Code civil et les principes de protection de la vie privée et du secret médical ont été adaptés en partant du présupposé qu'un protocole représente une tentative honnête et compétente de contribuer à la promotion de la santé et/ou au progrès de la connaissance. Cela n'est pas neutre.

\section{Conflit d'intérêt}

L'auteur déclare avoir aucun conflit d'intérêt.

\section{Remerciements}

L'auteur remercie les membres du CCTIRS et ses présidentes successives, Marie-Jeanne Mayaux et Catherine
Bonaïti-Pellié pour leur bon accueil, ainsi que Alain Leplège, Anne Fagot-Largeault et Joël Coste qui ont orienté ou accompagné la recherche doctorale dont cet article est issu. Il remercie également chaleureusement Émmanuèle Amoros pour ses commentaires sur une première version ainsi que deux relecteurs anonymes qui ont aidé à clarifier et préciser son propos.

\section{Références}

[1] Goldberg M. Cet obscur objet de l'épidémiologie. Sciences Sociales et Santé 1982;55-110.

[2] Ducimetière P. Épidémiologie et santé publique : poursuivre l'effort de clarification. Rev Epidemiol Sante Publique 1999;47:105-8.

[3] Ducimetière P. Recherche : la triple approche. Rev Epidemiol Sante Publique 2005;53:233-4.

[4] Buton F. De l'expertise scientifique à l'intelligence épidémiologique : l'activité de veille sanitaire. Genèses 2006;4:71-91.

[5] Dab W. L'aide à la décision en santé publique : problématique et enjeux. Après-demain. Journal mensuel de documentation politique 1990;320321:25-8.

[6] Salamon R. La formation en épidémiologie humaine à l'université. In: Valleron AJ, editor. L'épidémiologie humaine. Conditions de son développement en France et rôle des mathématiques. Paris: EDP Sciences; 2006. p. 287-99.

[7] Goldberg M, Salamon R. L'épidémiologie humaine. In: Valleron AJ, editor. L'épidémiologie humaine. Conditions de son développement en France et rôle des mathématiques. Paris: EDP Sciences; 2006 . p. 3970.

[8] Valleron AJ, Schwartz D. Définition historique et champs de l'épidémiologie humaine. In: Valleron AJ, editor. L'épidémiologie humaine. Conditions de son développement en France et rôle des mathématiques. Paris: EDP Sciences; 2006. p. 1-36.

[9] Amsterdamska O. Demarcating epidemiology. Science Technology \& Human Values 2005;30:17-51.

[10] Speers M. Guidelines for defining public health research and public health non-research (report). Centers for Disease Control and Prevention. Atlanta, Georgia. 1999. http://www.cdc.gov/od/ads/opspoll1.htm, accédé le 05/02/2006.

[11] Hodge JG, Gostin LO. Public health practice vs Research. A report for public health practitioners including cases and guidance for making distinctions. Rapport du Council of State and Territorial Epidemiologists/Centers For Disease Control and Prevention. Atlanta, Georgia, 2004. http://www.cste.org/pdffiles/newpdffiles/CSTEPHResRptHodgeFinal. 5.24.04.pdf, accédé le 13/03/2007.

[12] Lacey H. Is science value free? Values and scientific understanding. London, New-York: Routledge; 2005.

[13] Longino HE. Science as social knowledge: values and objectivity in scientific inquiry. Princeton: Princeton University Press; 1990.

[14] Coughlin SS. Ethics in epidemiology at the end of the 20th century: ethics, values, and mission statements. Epidemiol Rev 2000;22:169-75.

[15] Weed DL. The merger of bioethics and epidemiology. J Clin Epidemiol 1991;44:15-22S.

[16] Lechopier N. Ethique dans la recherche et démarcation. La scientificité de l'épidémiologie à l'épreuve des normes de confidentialité. Thèse de doctorat de philosophie des sciences. Paris: Université Paris-1 Panthéon-Sorbonne; 2007.

[17] De Lamberterie I, Lucas H. Informatique, libertés et recherche médicale. Paris: CNRS Éditions; 2001.

[18] Marliac-Négrier C. La protection des données nominatives informatiques en matière de recherche médicale. Aix-en-Provence: Presses Universitaires d'Aix-Marseille; 2001.

[19] Bonaïti-Pellié C, Arveux P, Billette de Villemeur A, Dantchev N, Elbaz A, Fabre-Guillevin E, et al. Traitement de l'information en matière de recherche dans le domaine de la santé : nul n'est censé ignorer la loi ! Med Sci 2009;25:93-8. 
[20] Claudot F, Alla F, Fresson J, Calvez T, Coudane H, Bonaïti-Pellié C. Ethics and observational studies in medical research: various rules in a common framework. Int J Epidemiol 2009;38:1104-8.

[21] Bara C, Berr C, Blacher J, Bloch J, Bonaiti C, Calvez T, et al. Projet de loi relatif à la protection des personnes physiques à l'égard des traitements de données à caractère personnel. Position du comité consultatif sur le traitement de l'information en matière de recherche dans le domaine de la santé (CCTIR). Rev Epidemiol Sante Publique 2003;51:364-7.

[22] Freedman B. Scientific value and validity as ethical requirements for research: a proposed explication. IRB. Rev Hum Subj Res 1987;9:7-10.

[23] Boltanski L, Thévenot L. De la justification. Paris: Gallimard; 1991.

[24] Kessler DA, Rose JL, Temple RJ, Schapiro R, Griffin JP, TherapeuticClass Wars. Drug promotion in a competitive marketplace. N Engl J Med 1994;331:1350-3.

[25] Sismondo S. Pharmaceutical manoeuvers. Social Studies of Science 2004;34:149-59.

[26] Hill KP, Ross JS, Egilman DS, Krumholz HM. The ADVANTAGe seeding trial: a review of internal documents. Ann Intern Med 2008;149:251-8.

[27] Sox HC, Rennie D. Seeding trials: just say « No ». Ann Intern Med 2008;149:279-80.

[28] Rouquette C, Schwartz D. Méthodes en épidémiologie. Paris: Flammarion; 1970.

[29] Rumeau-Rouquette C, Bréart G, Padieu R. Méthodes en épidémiologie. Échantillonnage investigations analyse. Paris: Flammarion MédecineSciences; 1985.

[30] Schwartz D. À quoi sert l'épidémiologie ? La Revue du Praticien 1982;32:33:2209-18.

[31] Hempel CG. Eléments d'épistémologie. Paris: Armand Colin; 2002.
[32] Rothman KJ, Greenland S. Validity and generalizability in epidemiologic studies. In: Armitage P, Colton T, editors. Encyclopedia of biostatistics. Chichester, New York: J. Wiley; 1998. p. 4700-6.

[33] Berlivet L. Controverses en épidémiologie. Production et circulation de statistiques médicales. Ministère de l'Emploi et de la Solidarité, Direction de la recherche des études de l'évaluation et de la statistique (DREES), Programme épidémiologie gestion des risques gestion des services, 1995. (Convention MiRe no. 11-94 1995).

[34] Berlivet L. Exigence scientifique et isolement institutionnel : l'essor contrarié de l'épidémiologie française dans la seconde moitié du 20è siècle. In: Jorland G, Opinel A, Weisz G, editors. Body counts. Medical quantification in historical and sociological perspectives. Montréal: McGill-Queen's University Press; 2005. p. 335-58.

[35] Gaudillière J. Inventer la biomédecine : la France, l'Amérique et la production des savoirs du vivant (1945-1965). Paris: La Découverte; 2002.

[36] Bourdieu P. Science de la science et réflexivité. Paris: Raisons d'agir; 2001

[37] Lechopier N. Confidentialité et confiance. L'émergence de normes pour la recherche épidémiologique. In: Martin T, Quiviger PY, editors. Action Médicale et Confiance. Besançon: Presses universitaires de FrancheComté; 2007. p. 307-30.

[38] Fagot-Largeault A. Autonomie don et partage dans la problématique de l'expérimentation humaine. Dialogue 1991;30:355-63.

[39] ADELF. Déontologie et bonnes pratiques en épidémiologie Rev Epidemiol Sante Publique 1999; 47:1S-32S.

[40] Lechopier N. L'émergence de normes pour la recherche biomédicale. À l'origine de la loi Huriet (1975-1988). Med Sci 2004;20: $377-81$. 